\title{
Norois
}

Environnement, aménagement, société

205 | 2007/4

Vivre les espaces périurbains

\section{La diversité des modes d'habiter des espaces périurbains dans les villes intermédiaires : différenciations sociales, démographiques et de genre}

The ways of living diversity in the peri-urban spaces of French intermediate towns: social, demographic and gender differentiation's

\section{Laurent Cailly et Rodolphe Dodier}

\section{(2) OpenEdition}

Journals

Édition électronique

URL : https://journals.openedition.org/norois/1266

DOI : $10.4000 /$ norois. 1266

ISBN : 978-2-7535-1553-6

ISSN : 1760-8546

Éditeur

Presses universitaires de Rennes

Édition imprimée

Date de publication : 1 décembre 2007

Pagination : 67-80

ISBN : 978-2-7535-0616-9

ISSN : 0029-182X

Référence électronique

Laurent Cailly et Rodolphe Dodier, «La diversité des modes d'habiter des espaces périurbains dans les villes intermédiaires : différenciations sociales, démographiques et de genre », Norois [En ligne], 205 | 2007/4, mis en ligne le 01 décembre 2009, consulté le 13 janvier 2022. URL : http:// journals.openedition.org/norois/1266; DOI : https://doi.org/10.4000/norois.1266 


\title{
LA DIVERSITÉ DES MODES D'HABITER DES ESPACES PÉRIURBAINS \\ DANS LES VILLES INTERMÉDIAIRES : DIFFÉRENCIATIONS SOCIALES, DÉMOGRAPHIQUES ET DE GENRE
}

\author{
Laurent CailLy \\ COST - UMR CITERES 6173 CNRS \\ (Université François-Rabelais), \\ 33 allée Ferdinand-de-Lesseps - 37000 Touns \\ laurent.cailly@univ-tours.fr \\ RODOLPHE DODIER \\ GREGUM - UMR ESO 6590 CNRS \\ (Université du Maine), \\ avenue Olivier-Messiaen - 72085 Le MANS cedex 9 \\ Rodolphe.Dodier@univ-lemans.fr
}

\begin{abstract}
RÉSUMÉ
Les espaces périurbains des villes intermédiaires pewvent sembler, en première lecture, de faible diversité sociale. Pourtant, ils sont traversés par plusieurs plans de différenciations, gradient proche couronne/périurbain lointain, quadrants internes à l'aire urbaine, segments du marché immobilier, qui correspondent à autant de facteurs de diversité des modes d'habiter. Partant d'acquis antérieurs et basés sur environ 60 entretiens auprès d'habitants de la périphérie de Tours et du Mans, cet article se propose d'explorer les différenciations dans les modes de vie périurbains selon l'itinéraire résidentiel, l'appartenance sociale et les différenciations socio-démographiques (âge et genre). La trajectoire résidentielle et à travers elle l'expérimentation de contextes socio-résidentiels, est sans doute l'une des variables les plus discriminantes. Le parcours antérieur conditionne une plus ou moins grande adhésion au modèle pavillonnaire. Les différenciations sociales restent structurées par une opposition entre habitus populaire et habitus cultivé. La mobilité non contrainte et son pendant, le repli sur le logement, ainsi que le rapport à la ville sont à la base du maintien d'un fort effet de position sociale. Les différenciations socio-démographiques selon l'âge sont également importantes, induisant des plans de clivage au sein même des familles, notamment entre parents et grands adolescents, qui sont les plus critiques vis-à-vis d'une localisation résidentielle dans le périurbain. Enfin, le partage des taches à l'intérieur des couples produit aussi des représentations différenciées et des rapports à la ville, au village périurbain et au logement variées entre hommes et femmes, selon la plus ou moins grande conformation au modèle traditionnel de la famille ou au modèle du couple égalitaire.
\end{abstract}

Mots Clé : Périurbain, Modes d'habiter, Mobilité, Groupes sociaux. 


\section{ABSTRACT \\ The ways of living diversity in the peri-urban spaces of French intermediate towns: social, demographic and gender differentiation's}

At first glance, the peri-urban spaces of French intermediate towns seems little diversified on a social plane. However, they are divided into an important number of areas i.e.: near and far peripheries, quadrants, residential areas. These residential segregations generate important differentiations regarding ways of living. Based on previous works and on about sixty investigations carried out in the peri-urban peripheries of Le Mans and Tours, this article zooms on the differentiation of practices and space representations, according to residential trajectory, social position, age and sex. The residential trajectory is strongly discriminating because the adherence to the peri-urban way of life is conditioned by the past experiences. The social differences, which are mainly structured by an opposition between working classes habitus and cultured habitus, are pronounced about house relationship, leisure mobility and representations of town. The age parameter plays an important part as it induces diversity between the family members i.e. between parents and old teenagers: these are generally critical with the peri-urban residential choice. Lastly, the division of labour within the couple explains the differences between men and women about practices and representations of house, peri-urban village and town: some of them adhere to the traditionally model of family when the other adhere to the egalitarian model.

KEY WORDS: peri-urban, ways of living, mobility, social groups.

Appréhendés à petite échelle, les espaces périurbains des villes intermédiaires semblent assez peu diversifiés. Privés des extrêmes - ni très riches, ni très pauvres -, ils constituent, par excellence, le territoire des classes moyennes (Jaillet, 2004), cet ensemble composite et particulièrement large qui, de l'ouvrier qualifié au cadre, a bénéficié du régime d'accumulation fordiste, atteint un certain niveau de solvabilité et donc pu accéder à la propriété en maison individuelle. Liés à une étape dans le cycle de vie - la venue au monde des enfants, la fondation du «foyer », l'achat d'une maison individuelle - les espaces périurbains constituent également le territoire des familles, des parents et des enfants, engagées dans la réalisation d'un projet d'existence fondamentalement patrimonial. Les modes de vie qu'ils inventent paraissent dans leurs grands traits relativement homogènes : pavillons, jeux d'enfants et barbecues dans les jardins, fort investissement domestique, bi-motorisation, navettes quotidiennes vers la ville-centre, pratique privilégiée des centres commerciaux périphériques, sociabilités néo-villageoises mythifiées, etc. Pourtant, cette apparente uniformité des espaces et du quotidien périurbains ne résiste pas vraiment à l'investigation scientifique. En réalité, elle masque bien des lignes de partage qui procèdent tout à la fois de la diversité des trajectoires spatiales, des contextes sociaux et démographiques, et par inférence, des modes d'habiter.

Ce travail se situe dans la lignée des approches géographiques renouvelées autour de la question de l'habiter (voir par exemple le numéro spécial 115-118 de la revue TIGR). Toutefois, l'acception d'habiter que nous retenons est moins celle d'une manière globale d'être-au-Monde (Hoyaux, 2002) qu'un ensemble d'attitudes et de pratiques spatiales, d'abord fondées sur des temporalités quotidiennes, et visibles en particulier à travers les rapports au logement, à un espace local - quartier en ville (Authier, 2001), village dans le périurbain - et à l'ensemble de la ville ou de l'espace métropolitain. L'attention est en fait plus portée sur les modes d'habiter, c'est-à-dire sur l'ensemble des dispositions et des pratiques qui régissent les rapports à l'espace, la façon d'être mobile ou les identités spatiales afférentes. Ce travail se situe aussi dans un contexte d'interrogation croissante autour des espaces périurbains, qui se traduit autant par des mises en commun fécondes de travaux (GIS socio-économie de l'habitat), que par des écrits sur leur supposé déficit d’urbanité (Lévy, 2003) ou encore sur l'émergence d'un tiers-espace (Vanier, 2005). 
Les deux recherches dont nous allons proposer les résultats, bien que menées séparément et différemment au Mans et à Tours, présentent des résultats convergents : la forte différenciation des identités spatiales des périurbains quant à leurs rapports au logement, à l'espace local (village périurbain) ou à la ville, atteste d'une fragmentation, et même d'une relative individualisation de la société périurbaine. Cependant, ce foisonnement ne doit pas aveugler. D'une part, il n'exclut pas le maintien de principes de structuration collective, de plans de clivages vigoureux en fonction de l'histoire résidentielle, de la position sociale, de l'âge ou du sexe. D'autre part, il est moins le produit des marges de manœuvre croissante dont on crédite les individus (Lévy, 2004) que l'effet de la subtile alchimie que génère le recoupement, à l'échelle individuelle, de ces différents contextes, qui explique que chaque habitant est toujours, au bout du compte, le dépositaire d'une spatialité singulière. Dans cet article, nous allons successivement examiner l'effet de la trajectoire résidentielle, de la position sociale, de l'âge et du genre sur la différenciation des modes d'habiter. Nous verrons combien ces contextes, bien que partiellement dépendants, expliquent pour une large part la diversité des modes d'habiter, par le jeu des combinaisons multiples.

Les conclusions livrées ci-dessous résultent de deux enquêtes menées de manière autonome au Mans (Dodier, 2006) et à Tours (Cailly, 2004). À Tours, le panel est composé de 30 individus dont la plupart ont en commun de travailler dans le secteur public hospitalier (du médecin à l'agent de service) mais résidant dans des types d'espaces différents. Composé d'un tiers d'hommes et de deux tiers de femmes, ces personnes ont été longuement interrogées au minimum deux fois, sur leur parcours résidentiel, leurs pratiques spatiales (mobilité, travail, pratiques commerciales, culturelles, sportives, etc.) ainsi que sur leurs réseaux de sociabilité. Ces entretiens semi-directifs ont été enrichis par des carnets de pratiques (relevés de tous les déplacements sur 4 jours chômés et 4 jours travaillés). Au Mans, 35 ménages sont suivis depuis près de 5 ans, avec également plusieurs entretiens successifs sur les stratégies résidentielles et les pratiques spatiales. Pour certains ménages, les entretiens ont été menés avec les deux membres du couple, soit de façon concomitante, soit à des moments distincts, ce qui peut permettre d'éviter d'avoir des propos trop consensuels. Enfin, pour 8 d'entre eux, des entretiens autonomes ont été menés directement auprès des enfants, en particulier ceux âgés de 14 à 18 ans.

Centrées sur une analyse qualitative et compréhensive des pratiques et des représentations spatiales des habitants périurbains, ces enquêtes sont suffisamment proches dans la méthode, la démarche et l'objet pour que les résultats soient mis en commun et synthétisés, en s'inscrivant dans une logique de généralisation plutôt que dans une optique comparative. Le but n'est pas de comparer les espaces périurbains du Mans et de Tours, mais de montrer la pluralité des déterminants des modes d'habiter pour les habitants des espaces périurbains des villes intermédiaires.

\section{Diversité sociale et pluralité des modes d'habiter}

Dans les deux villes intermédiaires observées, la diversité sociale des espaces périurbains est un peu plus faible que celle des quartiers de la ville-centre car les catégories les plus aisées comme les catégories pauvres y sont moins représentées. Toutefois, derrière les effets de lissage engendrés par une lecture en termes de moyenne, tous les groupes sociaux sont présents. De fait, on retrouve en périphérie la même hétérogénéité qu'ailleurs dans la ville, zones sensibles et beaux quartiers exceptés. Cela tient à la diversité des contextes spatiaux que l'on peut regrouper sous le vocable de périurbain, mais aussi à la diversité, dans une couronne donnée, des profils sociaux des populations résidentes. Trois plans de différenciations spatiales expliquent une grande part des variations de composition sociale observées.

\section{FACTEURS DE DIFFÉRENCIATION SPATIALE}

Le premier plan de différenciation fonctionne, de façon classique, selon un gradient centrepériphérie. La première couronne périurbaine, ancienne, mieux desservie - donc très accessible 
- et bien équipée en services de proximité, est majoritairement composée de catégories moyennes supérieures cherchant à concilier maison individuelle, faibles densités urbaines et rapidité d'accès à la ville. Symétriquement, la pression immobilière et le renchérissement des prix dans les premières couronnes, conduit à l'éloignement des catégories populaires solvables qui se retrouvent massivement dans le périurbain lointain, c'est-à-dire dans les communes les moins accessibles et les moins équipées, y compris en infrastructures scolaires.

Un second plan de différenciation fonctionne selon des quadrants plus ou moins nets. Au Mans, la partie sud connaît un développement périurbain ancien, notamment sous l'impulsion des ouvriers travaillant chez Renault. Elle est donc plus populaire que le secteur nord-ouest, de développement plus récent, aux aménités environnementales plus importantes (zone de bocage et de petites forêts), et dans lequel les catégories moyennes supérieures dominent. À Tours, les coteaux de la Loire, du Cher ou de l'Indre, à fort capital paysager, attirent également des ménages aisés, alors que les gâtines et les varennes humides accueillent davantage les classes moyennes inférieures.

Un troisième plan de différenciation, interne à chaque commune, dépend des segments de l’immobilier. La composition sociale des lotissements varie selon la taille des parcelles et l'ancienneté des constructions; les maisons isolées (pavillons ou fermettes rénovées) accueillent les catégories sociales plus aisées; le bâti ancien de bourg est susceptible de loger des ménages plus modestes, aux situations professionnelles parfois précaires.

Ainsi, au-delà de l'apparente homogénéité des lotissements, les espaces périurbains des villes intermédiaires se caractérisent au plan résidentiel par leur hétérogénéité socio-spatiale, ce qui confirme les tendances observées par M. Berger (2004) en périphérie de Paris.

\section{DIVERSITÉ DES MODES D’HABITER}

La diversité sociale accompagne, et parfois recoupe, une réelle diversité des modes d'habiter. Si un fort rapport au logement est une constante assez générale liée à la surreprésentation des familles parmi les ménages, de fortes différenciations sociales apparaissent dans les manières d'utiliser les différentes pièces (existence d'une grande pièce à vivre) et d'utiliser les extérieurs. De même, le rapport à la commune périurbaine, comme celui au quartier en milieu urbain (Authier, 2001), est très hétérogène et varie entre la recherche d'un idéal communautaire villageois et un certain détachement, parfois un désenchantement lorsque le contrôle social semble trop fort. Selon les cas, les pratiques spatiales dans la commune s'échelonnent du relatif évitement aux sociabilités intenses, en passant par la situation intermédiaire définie par des usages peu impliquants : achats quotidiens, école, loisirs des enfants, etc. Enfin, le rapport à la ville-centre oscille entre attraction pour ses ressources et son urbanité et répulsion pour sa congestion et son anonymat.

Les modes d'habiter périurbains sont donc divers, composites et témoignent d'une forte individualisation des rapports à l'espace. Pourtant, par-delà cette hétérogénéité, on peut observer un certain nombre de régularités, de logiques de structuration collective, qui s'articulent selon quatre dimensions différentes : en fonction de la diversité des trajectoires résidentielles; selon la positon sociale, dans ses deux dimensions socio-économiques et culturelles; en fonction de la place de l'individu dans le cycle de vie; et enfin selon le genre.

\section{La trajectoire résidentielle : des itinéraires hétérogènes}

La variété des modes d'habiter s'explique en partie par la diversité des trajectoires résidentielles, lesquelles conditionnent des formes différenciées d'expérimentation urbaine. Les enquêtes montrent qu'à position sociale identique, par exemple au sein des classes moyennes inférieures, le mode d'habiter diffère sensiblement selon que la personne soit d'origine rurale, citadine ou, pour les plus jeunes (moins de quarante ans), périurbaine. Les périurbains issus des campagnes témoignent le plus souvent d'un rapport productif au jardin, d'un plus fort retranchement domestique, d'une appréciation fréquemment négative de la ville - perçue comme une menace, un danger, un 
front qui avance -, ville qu'ils pratiquent rarement et souvent de manière obligée : travail, courses, formalités, etc. Parmi eux, on peut grossièrement distinguer ceux qui, originaires de la commune ou des alentours, en portent la mémoire et sont inscrits dans ce qu'il reste des anciens réseaux locaux, et ceux qui, originaires d'ailleurs, tendent à vivre repliés sur le domicile dans un certain anonymat. À l'opposé de ce premier groupe, les périurbains d'origine citadine découvrent un territoire « rural » qu'ils réinventent à travers l'usage ludique et ornemental du jardin, le bricolage domestique, l'expérimentation des promenades champêtres, les sociabilités néo-villageoises qu'ils cherchent parfois, au moins au départ, à reconstruire. Si la ville qu'ils disent fuir les ennuie, ils n'ont pas peur d'elle, en connaissent les ressources (commerciales et conviviales) et n'hésitent pas à les valoriser, même si c'est souvent à travers les centralités périphériques. Enfin, une dernière catégorie émerge parmi les jeunes adultes : les natifs du périurbain. Ceux-là tendent à reproduire le mode d'habiter transmis par leurs parents. Très attachés au modèle pavillonnaire, rompus aux déplacements automobiles, coutumiers des centres commerciaux, peu sensibles à la ville classique, ce sont eux, les périurbains de la deuxième génération, les plus fidèles porteurs du modèle d’urbanité périphérique.

La trajectoire résidentielle n'intervient pas que dans la différenciation des classes moyennes inférieures. Les enquêtes menées auprès de deux médecins périurbains tourangeaux, aux caractéristiques socio-démographiques identiques, ont montré que la proximité socio-spatiale n'engendre pas nécessairement la même manière d'habiter car celle-ci, avant toute chose, est largement tributaire de l'histoire résidentielle. Le premier médecin a toujours vécu en centre ancien, notamment dans le centre de Paris. Le second a principalement vécu en banlieue parisienne, excepté une courte période en zone dense vécue négativement. Résidant désormais dans le même type d'espace, ces deux individus revendiquent des identités spatiales bien distinctes - l'un se dit citadin, l'autre s'affirme banlieusard -, qui occasionnent deux types divergeant de spatialités. Le premier habite une fermette restaurée, vit la périphérie comme un retour à la nature mais sans rupture avec la ville dense (le centre de Tours, Paris) qui est grandement pratiquée (pour le travail, le commerce, les convivialités, les activités culturelles) grâce à une mobilité intense. Le second habite en pavillon et vit la périphérie (et le jardin) comme un refuge à l'écart de la ville; il est moins sensible aux charmes de l'urbain central, plus attiré par les galeries marchandes et l'urbanisme périphérique. Cet exemple montre bien que l'histoire résidentielle de l'individu, l'ensemble de ses habitudes et de ses goûts spatiaux socialement acquis, constitue le plus solide déterminant, parfois avant même la position sociale, des modes d'habiter.

\section{Le maintien des effets de position sociale}

L'appartenance sociale reste cependant un facteur important de différenciation dans les modes de vie. Dans les aires urbaines de Tours et du Mans, l'opposition entre un périurbain proche, ancien et bien équipé et un périurbain récent, peu équipé et plutôt populaire est d'ailleurs redoublée par une forte différenciation sociale des modes d'habiter. Cette césure s'exprime surtout à travers la mobilité hors-travail et le rapport à la ville.

\section{UNE MOBILITÉ TRÈS INÉGALE}

Les périurbains à faible niveau de ressources économiques et culturelles, appartenant aux classes moyennes inférieures, se caractérisent par une mobilité hors-travail réduite, par un très fort investissement du logement et de l'espace situé dans sa périphérie immédiate, commune ou petit pays. Parmi ceux-ci, on distingue deux principaux archétypes. Le « reclus » se caractérise par une très faible mobilité hors-travail (limitée aux déplacements contraints) et par un investissement important du logement. L'espace domestique constitue un havre dans lequel il se complaît, sort difficilement et passe l'essentiel de son temps. Cette réclusion ne procède pas seulement d'un manque de ressources mais aussi d'un système d'appétences parmi lesquelles on trouve le goût 
pour les travaux ménagers, le bricolage, le jardinage, etc. Bien souvent, elle découle d'une logique sécuritaire et traduit un réflexe défensif. Le «villageois » investit généralement beaucoup son logement mais, à la différence du « reclus », n’y vit pas replié. Sa mobilité hors travail, parfois forte, s'inscrit localement, autour du domicile, dans un espace réduit à la commune de résidence et aux communes voisines. Le cœur de l'agglomération est évité. Les activités de loisirs, les relations sociales, mais aussi l'attachement à la localité font naître ainsi, à la marge de la ville, un petit «pays ». Cette propension à l'ancrage et à la territorialisation locale, dans les espaces périurbains, semble plutôt caractéristique d'un habitus " populaire » (Cailly, 2004) et est reliée à d'autres dispositions sociales telles que la faible citadinité, le grégarisme familial ou l'enracinement résidentiel.

À l'inverse, les périurbains aisés, surtout lorsqu'ils disposent d'un fort capital scolaire, se distinguent par une mobilité hors-travail intense qui se déroule, pour une bonne part, à l'échelle de l'aire urbaine - dont ils consomment un grand nombre de ressources - ou vers d'autres métropoles et, au premier chef, Paris. Ces périurbains manifestent donc une meilleure capacité à articuler toutes les échelles, du domicile aux grandes villes et une spatialité principalement rhizomique qui n'exclut pas des formes, très fragmentaires, de territorialisation (la commune de résidence, le centre-ville de Tours, un quartier de Paris, etc.). Au sein des classes moyennes supérieures périurbaines (médecins, enseignants, ingénieurs) cette figure du «métropolitain » est la plus représentée. Comme les autres, celui-ci est attaché à son logement et manifeste parfois un certain localisme. Toutefois, il se distingue radicalement des autres par sa capacité à maîtriser et à valoriser positivement, pour ses activités culturelles, sportives ou ses sociabilités, particulièrement denses, l'échelle de l'aire urbaine et, parfois, les gisements d'urbanité que présentent les villes de rangs supérieurs. Pour ces périurbains, contrairement aux précédents, la mobilité n'est pas une contrainte mais constitue un principe d'épanouissement, parfois même une forme de distinction. Cet inégal rapport à la mobilité en terme d'intensité, de formes et d'échelles recoupe une seconde ligne de partage.

\section{UNE INÉGALE CITADINITÉ}

La pratique et la représentation de la ville, en particulier du centre, constitue cette deuxième source de différenciation de la société périurbaine, en étroite relation avec le capital culturel. Malgré un choix résidentiel périphérique souvent animé par un désir de nature, les plus qualifiés revendiquent généralement une forte appétence citadine, c'est-à-dire un goût pour la ville dense, pédestre et pour les lieux à forte urbanité. Ils apprécient la ville pour ses ambiances sensibles, son atmosphère, ses ressources architecturales et patrimoniales, pour les lieux culturels qui s'y trouvent (et qu'ils fréquentent) et pour le commerce spécialisé. De fait, ils valorisent nettement, à travers leurs pratiques commerciales, culturelles et festives, comme dans leurs discours, le centre historique de l'agglomération, de la même manière qu'ils valorisent les centres des grandes villes et, en particulier, le centre de Paris. À l'inverse, ils tendent à minimiser leurs pratiques des centralités périphériques et à décrier fortement ce type d'espace qu'ils qualifient volontiers d'impersonnels, de froids, d'inesthétiques, voire d'emblématiques de la société de consommation. Ces périurbains cumulent donc, grâce à leur mobilité intense, exurbanisation résidentielle et fort rapport à la ville.

À l'inverse, les individus appartenant aux classes moyennes inférieures périurbaines développent beaucoup plus fréquemment des idéologies anti-urbaines. Ces idéologies anti-urbaines s'expriment d'abord sur un plan pratique (difficultés d'accès, de stationnement), se déclinent ensuite sur la base du rapport aux autres (anonymat, foule) et peuvent dans certains cas prendre un style plus défensif voire sécuritaire (peur des agressions). De fait, en dehors du travail, ces périurbains évitent autant que possible un cœur d'agglomération qu'ils réprouvent. Du coup, ils tendent à valoriser les centralités périphériques, dans lesquelles ils peuvent passer parfois beaucoup de temps. Cette disposition anti-urbaine (c'est-à-dire ce dégoût affiché pour les lieux à forte urbanité) peut procéder 
d'une continuité biographique, lorsqu'elle concerne des personnes d'origines rurales venues travailler en ville mais qui ont une faible expérience ou habitude de la ville. Toutefois, elle concerne aussi des personnes qui ont longtemps vécu en ville, qui ont une solide expérience de la ville et eu un réel goût pour la ville, mais que l'exurbanisation (souvent contrainte) a en quelque sorte « décitadinisé ». Dans ce cas, c'est l'adoption du modèle pavillonnaire périphérique qui entraîne assez mécaniquement l'expression d'idéologies anti-urbaines.

Ainsi, dans les périphéries de Tours et du Mans, il apparaît que plus les moyens et la compétence culturelle des habitants baissent (et, généralement, plus on s'éloigne de l'agglomération), plus le rapport à la ville se distend, plus la mobilité hors-travail s'affaiblit, plus le repli sur l'espace local ou domestique est marqué et associé à des idéologies anti-urbaines. Toutefois, cette « loi », relativement binaire, supporte bien des exceptions, ne serait-ce que parce que bien d'autres éléments que la position sociale font varier le mode d'habiter, telle la position dans le cycle de vie.

\section{Cycle de vie et perception de l'espace périurbain}

Au sein même des ménages, les rapports au logement, au village périurbain ou à la ville varient fortement selon les individus. Les contraintes induites par l'activité professionnelle ou par l'activité scolaire pèsent sur la perception des différents espaces. Les possibilités de relations sociales dans l'espace résidentiel ou dans d'autres environnements sociaux ne sont également pas identiques selon l'âge des personnes. De fortes différenciations existent donc, formant parfois de véritables plans de clivage au sein des familles. Les jeunes enfants semblent s'épanouir dans l'espace périurbain mais la tendance sécuritaire tend à rendre leur autonomisation délicate. Sur ce point, nous manquons de données et cela nécessite des investigations complémentaires. Les grands adolescents sont clairement les plus critiques vis-à-vis d'une localisation résidentielle dans le périurbain, surtout lointain, où les difficultés liées à l'absence de mobilité autonome sont maximales.

\section{JEUNES ENFANTS DANS LE PÉRIURBAIN}

S'agissant de familles avec un ou plusieurs enfants, les pratiques spatiales de chaque individu évoluent en fonction de l'âge des uns et des autres, en particulier en fonction des relations sociales des différents membres du ménage. Les jeunes enfants du périurbain, lorsque les parents respectent la carte scolaire, présentent le plus souvent une territorialité locale. L'école, les activités sportives ou les activités culturelles localisées sur la commune de résidence ou une commune voisine, favorisent les sociabilités situées dans un rayon proche. Leurs pratiques spatiales se limitent donc à la commune périurbaine, éventuellement à quelques communes contiguës à travers les activités de loisirs, culturelles ou sportives.

Les pratiques spatiales des enfants sont très encadrées, les parents les emmenant à l'école ou chez l'assistante maternelle et les récupérant le soir, les conduisant vers leurs lieux de loisirs et les récupérant une ou deux heures plus tard dès la fin de l'activité. Le seul espace où ils circulent de façon autonome est celui de la proximité immédiate, c'est-à-dire l’impasse dans les lotissements, le segment de rue si elle est peu passante, et les quelques maisons dans lesquelles des voisins ont des âges en rapport. À la différence des autres, les enfants du périurbains sont probablement plus attachés à leur résidence - le jardin constituant un espace privilégié de jeu -, sont relativement plus contrôlés et moins autonomes ne serait-ce que parce que les équipements (jeux, bibliothèque, piscine) sont à distance. Il est parfois délicat de les laisser circuler seuls sur leur commune car l'absence de trottoir, de pistes cyclables ou d'éclairage pose problème. De fait, pour leur mobilité, ils sont souvent tributaires de leurs parents.

L'argumentaire sur cet encadrement étroit tourne souvent autour de la sécurité, terme polysémique pouvant signifier aussi bien la crainte de la rencontre d'un pédophile que des aspects de sécurité routière. Le sentiment sécuritaire nuit sans doute à l'autonomisation des jeunes enfants, mais cette territorialité de proximité liée à l'enfance se retrouve ailleurs dans la ville. Pour autant, 
la territorialité locale des enfants est loin d'être systématique, d'une part car beaucoup de parents (surtout pour les familles aisées) contournent la carte scolaire et scolarisent leurs enfants dans le centre; d'autre part, parce que les familles cultivées favorisent d'une manière ou d'une autre l'accès à l'ensemble des ressources métropolitaines (spectacles, cinémas, piscine, activités rares, etc.).

\section{ÊTRE ADOLESCENT DANS LE PÉRIURBAIN}

Les adolescents, parfois à l'entrée au collège, plus sûrement à l'entrée au lycée, voient subitement leur quotidien réorienté vers la ville et leurs pratiques spatiales s'élargissent considérablement. La scolarité est à la base des pratiques spatiales, induisant un départ matinal par le car scolaire, la découverte d'itinéraires nouveaux dans une petite ville périurbaine ou dans un quartier périphérique de l'agglomération voisine. Selon la carte scolaire, cette « entrée en ville » peut être différenciée. Certains secteurs périurbains mènent à des collèges ou à de grands lycées suburbains : dans ce cas, l'acheminement par le car ou en voiture à la porte de l'établissement et la faible urbanité du quartier ne remettent pas foncièrement en cause le mode de vie périphérique. Dans d'autre cas, par le recours aux collèges ou aux lycées privés dans le cadre de stratégies d'évitement scolaire, ou parce que le secteur scolaire mène aux établissements de centre-ville, l'expérimentation de la ville est beaucoup plus intense. Il s'y forme de nouvelles sociabilités, certes contraintes par les horaires scolaires, mais dont les jeunes s'affranchissent de plus en plus au moyen du téléphone portable, à la fois facteur de contrôle par les parents et moyen d'émancipation.

L’entrée au lycée marque d'ailleurs une nouvelle étape, avec la possibilité accordée de sortir de l'enceinte scolaire avant l'heure du car et la découverte du centre de la grande ville. Dans ce cas, il est certain que cela favorise une certaine ouverture citadine ne serait-ce que parce que le centre-ville devient un point obligé ou exerce une séduction. Des amitiés se nouent entre adolescents périurbains et adolescents du centre. Les rues commerçantes, les cafés, les cinémas peuvent devenir le support du loisir et des sociabilités lycéennes. L'espace des loisirs et des sociabilités s'élargit donc, alors que les liens avec le village périurbain se distendent, ainsi que les liens avec le logement (excepté leur chambre). Cette nouvelle identité spatiale entre en conflit, au propre comme au figuré, avec celle des parents, surtout si ceux-ci ont une pratique distante de la ville. Le lycéen périurbain peut demander à retourner au centre-ville le soir ou le week-end. La question de la mobilité pour les loisirs et les sociabilités se pose donc rapidement avec acuité et est souvent un facteur premier de conflit dans la famille. Ce sont donc les grands adolescents qui souffrent le plus de l'éloignement de la ville, la vie de leur groupe social ayant lieu dans le centre ou dans la proche périphérie (multiplexes, etc.) ou bien se résume à «glander sous l'abribus » dans la commune périurbaine.

De plus, les « jeunes » apparaissent comme un groupe aux modes de vie très segmentés, reproduisant en grande partie les différenciations de l'ensemble de la société, notamment les différenciations de genre et d'origine sociale. Ils sont confrontés à une société locale qui n'est pas conçue pour eux, à des perceptions déformées ou trop généralisantes, et à une véritable prescription spatiale, c'est-à-dire que ce sont d'autres groupes sociaux qui leur affectent une place dans les villages périurbains.

Pour ces adolescents, le fait de résider dans l'espace périurbain entraîne une distorsion forte entre pratiques rêvées et pratiques spatiales réelles. Les difficultés de mobilité conduisent à une forte envie "d'ailleurs ", très souvent traduite en envie de ville. On peut d'ailleurs se demander si les espaces périurbains ne fabriquent pas des urbains? Cette question mérite d'être posée, parce que les lieux de scolarisation, et/ou de loisirs et de socialisation, puis d'insertion professionnelle et de migration résidentielle, sont ou seront, pour les jeunes périurbains, essentiellement des espaces urbains. Ils apprennent progressivement les codes et les valeurs des modes de vie urbains, les assimilent et les reproduisent tout en vivant plus ou moins bien leur présence dans le périurbain. Par la frustration initiale ou par transmission familiale, ils peuvent devenir des ambassadeurs de l'urbanité au moins aussi enthousiastes que des urbains d'origine. 
D'autres aspects de la position dans le cycle de vie restent à analyser, notamment le rôle, pour les adultes actifs, du passage d'une activité professionnelle urbaine à une retraite plus centrée sur la commune de résidence. Le vieillissement sur place des premières générations de périurbains commence ainsi à poser de nouveaux problèmes d'accessibilité aux services et engendre la diffusion de nouveaux besoins (Luxembourg, 2005). La position dans le cycle de vie implique donc des disparités dans la manière de percevoir les différents types d'espaces et dans les pratiques spatiales concrètes, en particulier entre jeunes enfants, grands adolescents et parents.

\section{Différenciations de genre}

Les différenciations de genre sont également marquées, quoique moins univoques. Le partage des tâches à l'intérieur des couples reste très inégal, en particulier au niveau des tâches induisant une mobilité, qui sont au cœur de nos interrogations. Cela génère des représentations différenciées de la mobilité, globalement mieux acceptée par les hommes, voire perçue comme valorisante. L'investissement affectif dans le logement est assez important pour les hommes comme pour les femmes mais prend des formes différentes. Les liens avec le village périurbain sont plutôt assurés par les femmes même si les exceptions sont nombreuses. Enfin, le rapport à la ville est plus homogène, correspondant plus à des différenciations selon la position sociale, bien que les pratiques puissent être différenciées. Globalement, les différenciations observées entre ménages correspondent assez bien au positionnement social, au moins dans les discours, et varient selon la plus ou moins grande conformation du ménage au modèle traditionnel de la famille ou au modèle du couple égalitaire.

\section{PERCEPTION DE LA MOBILITÉ ET RAPPORT AU TRAVAIL}

Anne, employée dans la fonction publique territoriale à mi-temps dans une commune voisine et Rémy, technico-commercial basé au Mans, résident depuis 8 ans à $20 \mathrm{~km}$ au nord du Mans avec leurs deux garçons (10 et 8 ans). Le partage des tâches au sein de leur couple est très clair, induisant des pratiques spatiales nettement différenciées: Anne s'occupe des enfants, les emmène à l'école en semaine et au sport le mercredi, s'occupe du ménage de la maison, des courses quotidiennes ou hebdomadaires dans la grande surface périphérique la plus proche. Rémy, avec ses journées de plus de 10 heures, est peu présent dans le logement en semaine, mais le samedi, il va chercher les garçons à l'école, tond la pelouse et bricole dans la maison. Anne fait partie du club de gymnastique volontaire local, au sein duquel elle est membre du comité, s'investit aussi dans l'association des parents d'élèves et connaît beaucoup de monde dans la commune. Rémy fait un jogging au Mans le dimanche matin avec deux amis qui n'habitent pas la commune.

Ce ménage peut apparaître légèrement caricatural, mais c'est un bon archétype d'un type de répartition des tâches et de pratiques spatiales assez courant dans le périurbain, notamment pour les contraintes ou les loisirs qui impliquent une mobilité. Le rapport à la mobilité joue un rôle important et diffère significativement selon le genre. Si les hommes ont tendance à ne pas exprimer de difficultés liées à la mobilité quotidienne, voire parfois à valoriser ces moments comme des temps agréables, les femmes ont tendance à vivre cette mobilité de façon plus négative. Cela est également induit par leur participation plus importante à la mobilité de proximité, pour faire les courses, conduire les enfants à l'école ou ailleurs, bref à une mobilité que l'on peut qualifier de « contrainte », par la répartition des tâches à l'intérieur du couple, même si ce n'est pas toujours en ces termes qu'elle est présentée.

Pour les autres activités, la diversité interindividuelle est particulièrement importante. Globalement, il est difficile d'affirmer qu'avoir des activités en dehors du logement soit marqué par de fortes différenciations de genre. Par exemple, si un des membres du couple a des activités associatives, il est fort probable que ce soit aussi le cas pour l'autre. Toutefois, ce ne sont pas les mêmes activités qui sont pratiquées et, surtout, la dimension spatiale et la mobilité ne jouent pas 
de la même façon. Les femmes ont plus tendance à avoir des activités dans une relative proximité, incluant quelques communes des alentours, par contre les hommes hésitent moins à participer à des activités sur l'ensemble de la ville. Par contre, la fréquentation du centre-ville, de plus en plus évité par les ménages périurbains, reste le fait de certaines femmes des catégories sociales plutôt élevées (pratiques de chalandise spécifiques, lieu de relation sociale, etc.).

Le premier facteur de différenciation entre hommes et femmes se situe cependant au niveau du travail. Les choix résidentiels tiennent compte de la localisation à l'instant « $\mathrm{t}$ » des lieux de travail respectifs. Lorsque ceux-ci sont proches l'un de l'autre, cela influe peu sur la localisation résidentielle mais lorsque les deux conjoints ont des lieux de travail éloignés, la stratégie des ménages consiste généralement à minimiser les déplacements de la femme, anticipant sur le partage des tâches domestiques et la gestion des enfants. Lorsque les deux emplois sont contraignants, les choix vont souvent dans le sens de la sauvegarde de la carrière du mari et, au contraire, de la mise entre parenthèses définitive, ou plus souvent énoncée comme temporaire, de l'activité professionnelle de la femme.

Dans le périurbain lointain, il existe d'ailleurs beaucoup de choix par défaut. Le faible apport financier du deuxième salaire compte tenu des frais de garde des enfants et du transport, la difficulté de trouver un moyen de garde des enfants compatible avec des horaires contraignants, l'impossibilité pour les moins qualifiées de retrouver un emploi après un licenciement, conduit certaines femmes à renoncer à toute activité professionnelle, avec la mise en place d'un processus d'enfermement social (Rougé, 2005). Par contre, dans la première couronne, les cas de femmes inactives ou à temps partiel semblent globalement beaucoup mieux vécus, résultant de choix plus positifs et assumés, exprimés en termes de réalisation de soi, de relativisation de la valeur travail, de choix de vie centré sur les enfants. La question ne se pose toutefois pas dans l'autre sens (libérer du temps professionnel de l'homme pour s'occuper du transport des enfants ou assurer le quotidien), montrant que certaines valeurs restent bien intériorisées par les ménages.

Les explications données par les ménages eux-mêmes sont de plusieurs types. La plus courante est une explication de type "naturaliste », surtout pour tout ce qui touche aux enfants (relations avec l'école, transports, éducation des plus jeunes). C'est aussi le cas dans d'autres domaines, notamment celui de la maison, où il est parfois considéré comme « normal » que ce soit la femme qui prépare les repas et l'homme qui fasse le « gros » entretien du pavillon. Ces explications fondées sur la naturalisation d'un partage inégal des tâches semblent particulièrement prégnantes dans les milieux populaires.

Un second ensemble d'explications fournies par les ménages se base sur les contraintes professionnelles. Mais les individus, femmes autant que les hommes d'ailleurs, oublient souvent que si dans un couple la femme a moins de contraintes professionnelles, cela résulte bien d'un choix qui n'est pas neutre. C'est d'ailleurs surtout la perception du caractère incontournable de ces contraintes professionnelles qui diffère.

Un troisième ensemble d'explications fait apparaître toute la diversité des rapports de couple. Ainsi, dans certains ménages, la répartition des tâches est quelque chose de réfléchi, qui fait l'objet de choix et de négociations, avec parfois des fréquences quotidiennes. Bien sûr, il existe une justification a posteriori, lors des entretiens, mais la répartition des tâches, qui peut quand même être très inégalitaire, a fait l'objet de choix négociés. Dans d'autres ménages, la question n'est pas soulevée et c'est «naturellement » que se fait le partage des tâches, souvent avec une forte incidence pour la femme.

\section{MODES D’HABITER FÉMININS ET MASCULINS}

Le fort rapport au logement des périurbains est caractéristique des familles en général, autant que de ces types d'espace en particulier. Ce fort investissement dans l'espace du logement n'est pas marqué globalement par des différenciations de genre. Mais il est intéressant de constater que les modalités de ce rapport au logement peuvent être très différenciées entre hommes et femmes. 
La distinction porte essentiellement sur les tâches ménagères, très clairement majoritairement assurées par les femmes, et sur l'entretien des extérieurs (tonte, taille des haies) et les travaux importants dans la maison, qui restent encore l'apanage des hommes. Si, notamment en fonction des contraintes horaires de leur conjointe, quelques hommes préparent les repas, l'entretien courant de la maison est peu partagé, sauf exception. Par contre, on observe beaucoup plus de variété dans l'entretien courant de l'habitat et la décoration, ainsi que dans l'implication dans le jardin. Nous avons déjà montré que la nature des espaces verts environnant le pavillon (jardin potager ou d'agrément, fleurs, vergers, etc.) était un bon marqueur de l'adhésion à des valeurs urbaines ou campagnardes (Dodier, 2007). Or, ces activités ne semblent pas fondamentalement sexuées, étant assurées dans certains ménages par l'un ou par l'autre, sans dominante nette, et dans d'autres ménages par les deux conjoints simultanément. Dans ce second cas, c'est alors souvent décrit comme une activité qui permet de partager quelque chose dans le couple. Un ménage fait ainsi la description du ramassage et du brûlage des feuilles mortes (le terrain est boisé) comme étant un grand moment de «solidarité familiale », les enfants participant à la « corvée », apparemment avec beaucoup de plaisir.

Les rapports au village périurbain sont sans doute ceux qui sont le plus marqués par des différenciations de genre. C'est l'espace dans lequel les femmes s'investissent plus que les hommes, même s’il existe une importante diversité selon les ménages. Si, dans un couple, l'homme pratique beaucoup le village, il est presque certain que c'est aussi le cas de sa femme sauf exception particulière (cas d'un homme originaire du village, habitant dans la partie peu dense de la commune, dont la femme, médecin, fréquente peu le village). Par contre, l'inverse est beaucoup moins vrai, puisque dans de nombreux ménages, seule la femme est véritablement intégrée à la petite société locale. Cette intégration se fait avant tout par l'école, premier facteur de relations sociales entre familles périurbaines, qui est un domaine largement réservé aux femmes. Lorsque l'école primaire est ouverte le samedi matin, ce jour est une exception car les hommes sont nombreux à aller chercher leurs enfants, ce qui leur permet a contrario de mieux justifier (par les contraintes professionnelles...) leur absence les autres jours de la semaine. La représentation dans les conseils d'école est également plus masculine que la fréquentation moyenne des établissements, alors que l'accompagnement des sorties scolaires est presque qu'exclusivement féminine. Les déplacements des enfants pour des activités de loisirs sont également plutôt assurés par les femmes, jusqu’à la figure bien connue de la maman-taxi qui passe son mercredi à convoyer ses enfants. Certaines femmes déclarent ainsi réaliser jusqu'à 100 ou $150 \mathrm{~km}$ le mercredi, soit 8 ou 10 navettes, vers le petit centre local, vers les centres périphériques et parfois vers le cœur de l'agglomération. Ces activités jouent un rôle très important dans le besoin de temps libéré des femmes ainsi que dans la définition des rôles dans les couples périurbains. La participation de l'homme à ce type de transport est un assez bon indicateur de la conformation au modèle du couple égalitaire. Pour les associations locales, la participation des femmes ou des hommes dépend beaucoup de l'objet, les femmes étant sans surprise plus présentes dans les activités culturelles et sociales, les hommes dans les activités sportives. Les formes de sport-loisir (randonnée, etc.) et les associations festives sont les plus mixtes. Mais les femmes prennent de plus en plus des fonctions importantes dans les associations ainsi que des fonctions électives, notamment aux élections municipales de 2001 (Achin et Paoletti, 2002). De ce point de vue, l'évolution semble plus rapide dans les communes de la première couronne, pourtant plus importantes et donc plus lourdes à gérer, que dans les communes du périurbain lointain où le mouvement reste limité, en particulier en raison du mode de scrutin.

Le rapport à la ville est aussi marqué par des différenciations de genre, avec une tendance à une plus forte fréquentation de l'ensemble urbain par les hommes. C'est surtout le cas des ménages de deux actifs dans lesquels seul l'homme travaille dans la ville-centre où sa périphérie. Dans ce cas, la fréquentation de la grande ville reste très limitée pour la conjointe, essentiellement sous la forme d'une fréquentation des centres périphériques. L'inverse est toutefois également vrai, puisque pour reprendre l'exemple de la femme médecin, dans ce ménage c’est elle qui fréquente le plus la ville. 
Les contraintes professionnelles expliquent donc une grande part de ces différences. Le reste est constitué d'activités rares et surtout sportives, que les hommes pratiquent sans avoir peur de la mobilité qu'elles engendrent. Pour les relations sociales (amis d'enfance, anciens voisins, collègues, etc.), les déplacements sont partagés d'autant que les relations sociales prennent plus la forme de repas entre couples que de sorties dans le cadre d'un groupe d'amis. Pour les déplacements contraints (achats hebdomadaire dans un hypermarché, démarches administratives), il est difficile d'observer une véritable tendance sexuée, bien que parfois, seul un des conjoints assure ce rôle. Dans le domaine des relations à la ville, qui correspondent bien au statut social, les contraintes ou les opportunités socioprofessionnelles semblent constituer le principal facteur explicatif des différenciations interindividuelles observées.

\section{Conclusion}

L'expérience périurbaine peut donc être très différente à l'intérieur d'une même famille en fonction de l'âge et du genre. La position dans le cycle de vie détermine, pour les enfants, un élargissement progressif des pratiques spatiales. À l'inverse, pour les femmes, mais également pour les personnes qui cessent leur vie active, on observe le plus souvent une certaine rétractation de l'espace de vie. Les différenciations observées restent cependant fortement marquées par les différenciations sociales. Le problème de la mobilité des grands adolescents, par exemple, se pose avec plus d'acuité dans le périurbain lointain, plus populaire, parce que le coût financier de la mobilité est plus important, parce que le statut de l'enfant dans le ménage est moins valorisé, parce que les valeurs urbaines sont moins partagées par les parents. De même, les différences de pratiques spatiales liées au genre, en lien avec les types de rapports conjugaux - plus ou moins égalitaires - demeurent fortement influencées par la position sociale. En première couronne, au sein des classes moyennes supérieures, les éventuelles différences de répartition des tâches sont davantage discutées, négociées et apparaissent réversibles, même si la carrière professionnelle de l'homme reste souvent en position centrale dans la vie du couple. Par contre, dans le périurbain lointain, dans des situations sociales plus populaires, ces différenciations semblent plus souvent «naturalisées ».

Au final, la multiplicité des facteurs de différenciation et l'hétérogénéité des contextes individuels déterminent une large gamme de choix et de comportements spatiaux. Ce processus accrédite l'hypothèse d'une diversification des identités spatiales périurbaines. Cette pluralité s'exerce d'abord au niveau interindividuel, entre habitants des espaces périurbains. Si la position sociale des individus oriente fréquemment les deux autres «variables » (âge et sexe), et maintient certaines régularités, force est de constater que les différents plans de clivages ne sont pas toujours concordants et conduisent à une forte individualisation de la société périurbaine. De même, cette pluralité concerne l'individu lui-même, qui peut adopter plusieurs modes d'habiter, à la fois successivement, au fur et à mesure du déroulement de son itinéraire biographique, mais aussi simultanément, en étant à la fois ou tour à tour urbain ou périurbain dans ses modes d'habiter. Cette identité plurielle (Lahire, 1998) ressort à travers l'adoption d'identités narratives parfois différentes dans des entretiens successifs.

Cependant, reconnaître la pluralité des identités spatiales périurbaines ne revient pas à éluder les dimensions collectives. Certains classements collectifs, en particulier l'opposition entre un habitus populaire centré sur des valeurs familiales et villageoises et un habitus cultivé valorisant l'urbanité et des formes de distinction, conservent une certaine opérationnalité. Il existe même des processus cumulatifs entre appétences culturelles, compétences de mobilité et capacités financières, qui expliquent une bonne part des régularités spatiales observables. Les autres dimensions des différenciations sociales, position dans le cycle de vie ou effet de genre, sans être complètement inféodées à la dimension socio-économique et culturelle, participent souvent à sa production même. 
L'hétérogénéité intrinsèque des modes d'habiter périurbains et la prégnance des logiques d'individus recoupent, sans y être strictement calquée, les divisions observées au plan socio-résidentiel et permet de rompre définitivement avec l'image un peu simpliste d'un espace homogène, faute de diversité. Ce processus n'affaiblit pas la valeur heuristique du «périurbain » en tant que type d'espace, puisqu'à l'échelle d'une région urbaine, les espaces qu'il recouvre conservent une certaine spécificité, par opposition à d'autres types d'espace (central, péricentral, suburbain, etc.). Il semble qu'on ait tout à gagner à prendre cette pluralité des identités spatiales périurbaines au sérieux et à y voir une nouvelle source de questionnement concernant les enjeux sociaux (inégalités, justice socio-spatiale, droit à la ville) ou politiques (anticipation et résolution des conflits, définition des champs d'action, identification des catégories de publics) qui traversent les espaces périurbains ou qui y sont plus particulièrement lisibles.

\section{Bibliographie}

Achin (C.), Paoletti (M.), 2002. - « Le "salto" du stigmate. Genre et construction des listes aux municipales de 2001 », Politix, nº 60, p. 33-54.

Authier (J.-Y.) (dir.), 2001. - Du domicile à la ville, vivre en quartier ancien, Paris, Anthropos, 209 p.

Arlaud (S.), JeAn (Y.), Royoux (D.) (dir.), 2005. - Rural-Urbain, nouveaux liens, nouvelles frontières, Rennes, PUR, 506 p.

Baudelle (G.), Darris (G.), Ollivro (J.), Pihan (J.), 2004. - « Les conséquences d'un choix résidentiel périurbain sur la mobilité : pratiques et représentations des ménages », Cybergéo, n² 287, 17 p.

Berger (M.), 2004. - Les périurbains de Paris, Paris, CNRS, 317 p.

Cailly (L.), 2004. - Pratiques spatiales, identités sociales et processus d'individualisation - Étude sur la constitution des identités spatiales individuelles au sein des classes moyennes salariées du secteur public hospitalier dans une ville intermédiaire : l'exemple de Tours, thèse de doctorat, Université François-Rabelais de Tours, $459 \mathrm{p}$.

Charmes (E.), 2005. - La vie périurbaine face à la menace des gated communities, Paris, L'Harmattan, $219 \mathrm{p}$.

Dodier (R.), 2006. - « Habiter, ce que le périurbain nous apprend », Travaux de l'Institut de Géographie de Reims, n 115-118, daté 2003-2004, p. 31-44.

Dodier (R.), 2007. - « Quelle articulation entre identité campagnarde et identité urbaine dans les ménages périurbains? ", Norois, n² 202, 2007/1, p. 35-46.

Dodier (R.), 2007. - «Les périurbains et la ville, entre individualisme et logiques collectives », Annales de la Recherche Urbaine, $\mathrm{n}^{\circ} 102$, p. 31-40.

EstÈBE (P.), 2004. - «Quel avenir pour les périphéries urbaines? », Esprit, n 303, p. 82-95.

Guilluy (C.), NoyÉ (C.), 2004. - Atlas des nowvelles fractures sociales en France, Paris, Autrement, 64 p.

Haumont (N.), 2001. - Les pavillonnaires : étude psychologique d'un mode d'habitat, Paris, L'Harmattan, édition originale, 1966, CRU, L'Harmattan, 150 p.

Hoyaux A.-F., 2002. - « Entre construction territoriale et constitution ontologique de l'habitant : introduction épistémologique aux apports de la phénoménologie au concept d'habiter », Cybergéo, n 102, 18 p.

LAHIRE (B.), 1998. - L'homme pluriel, les ressorts de l'action, Paris, Nathan, 271 p.

JAILLET (M.-C.), 2004. - «L'espace périurbain : un univers pour les classes moyennes », Esprit, n³ 303, p. $40-60$.

Lévy (J.), 2003. - «Périurbain, le choix n’est pas neutre », Pouvoirs locaux, n 56, p. 35-42.

LÉVY (J.) (dir.), 2004. - SCALAB : les échelles de l'habiter, Rapport de recherche 548, PUCA, 335 p.

Luxembourg (N.), 2005. - Renouvellement social et immobilités en périurbain, le cas de l'aire métropolitaine marseillaise, Thèse de doctorat, Université Aix-Marseille III, 276 p.

Pinson (D.) ET Thomann (S.), 2001. - La maison en ses territoires, de la villa à la ville diffuse, Paris, L'Harmattan, $191 \mathrm{p}$. 
Rougé (L.), 2005. - Accession à la propriété et modes de vie en maison individuelle des familles modestes installées en périurbain lointain. Les « captifs » du périurbain?, Thèse de doctorat, Université de Toulouse 2, $381 \mathrm{p}$.

Semmoud (N.), 2003. - «L'habiter périurbain : choix ou modèle dominant? », Revue de Géographie Alpine, $n^{\circ} 91-4$, p. 57-64.

VANiER (M.), 2005. - « La relation ville/campagne excédée par la périurbanisation », Cahiers Français, n 328, p. 13-17.

Cet article a été reçu le le 4 juillet 2007 et définitivement accepté le 19 décembre 2007. 